\title{
Clinical characteristics and prognosis in oldest old patients with ischaemic stroke or transient ischaemic attack in China
}

\author{
Yu Wang ${ }^{1,2}$, Jing Jing ${ }^{1,2}$, Yuesong Pan ${ }^{1,2}$, Mengxing Wang ${ }^{1,2}$, Xia Meng ${ }^{1,2}$, Yongjun Wang ${ }^{1,2,3}$ \\ ${ }^{1}$ Department of Neurology, Beijing Tiantan Hospital, Capital Medical University, Beijing, China; ${ }^{2}$ China National Clinical Research Center for \\ Neurological Diseases, Beijing, China; ${ }^{3}$ Research Unit of Artificial Intelligence in Cerebrovascular Disease, Chinese Academy of Medical Sciences, \\ Beijing, China \\ Contributions: (I) Conception and design: Yongjun Wang, Yu Wang; (II) Administrative support: X Meng; (III) Provision of study materials or \\ patients: Yongjun Wang; (IV) Collection and assembly of data: Yongjun Wang, J Jing, X Meng; (V) Data analysis and interpretation: Yu Wang, J Jing, \\ Y Pan, M Wang; (VI) Manuscript writing: All authors; (VII) Final approval of manuscript: All authors. \\ Correspondence to: Yongjun Wang, MD, PhD. Department of Neurology, Beijing Tiantan Hospital, Capital Medical University, Beijing 100070, \\ China; China National Clinical Research Center for Neurological Diseases, Beijing, China; No. 119 South 4th Ring West Road, Fengtai District, \\ Beijing 100070, China. Email: yongjunwang@ncrend.org.cn.
}

Background: Limited data are available about the prognosis of ischaemic stroke or transient ischaemic attack (TIA) in oldest-old patients, especially in China. We aimed to describe the clinical characteristics and prognosis of oldest-old patients with ischaemic stroke or TIA in China.

Methods: Patients with acute ischaemic stroke (AIS) or TIA were recruited between August 2015 and March 2018 in the Third China National Stroke Registry (CNSR-III). Clinical characteristics including demographic data, medical history, medication use and stroke aetiology, were obtained. The outcomes were one-year stroke recurrence, combined vascular events (stroke, myocardial infarction, and cardiovascular death), mortality, and poor functional outcome [modified Rankin scale (mRS) 3-6]. Oldest-old was defined as $\geq 80$ years old. Clinical characteristics and prognosis were compared by different age groups $(<65,65-79$, and $\geq 80$ years). The association between age and prognosis was analysed using the multivariable Cox proportional hazards and logistic regression models.

Results: A total of 15,166 patients with AIS or TIA were included in this study with 929 (6.13\%) oldestold patients. Oldest-old patients had a higher likelihood of cardioembolic stroke or comorbid cardiac disease than other age groups. When compared with patients aged $<65$ years, oldest-old patients had higher risk of one-year stroke recurrence [adjusted hazard ratio (HR) 1.36; 95\% confidence interval (CI): 1.06-1.73, $\mathrm{P}=0.014$ ], combined vascular events [adjusted HR, 1.42; 95\% CI, 1.13-1.79, $\mathrm{P}=0.003$ ], mortality [adjusted odds ratio (OR), 4.25; 95\% CI: 2.99-6.04, $\mathrm{P}<0.001$ ] and poor functional outcome (adjusted OR, 4.25; 95\% CI: 3.40-5.33, $\mathrm{P}<0.001)$ with $\mathrm{P}$ for trends $<0.001$ among age groups.

Conclusions: Oldest-old patients differed from younger patients regarding clinical characteristics, stroke aetiology, and secondary preventive medication persistence with a poor clinical prognosis in China. Current information on profile of the oldest-old stroke patients is crucial to develop specific secondary prevention and treatment strategies.

Keywords: Ischaemic stroke; transient ischaemic attack (TIA); elderly; prognosis

Submitted Sep 30, 2021. Accepted for publication Feb 10, 2022.

doi: 10.21037/apm-21-2830

View this article at: https://dx.doi.org/10.21037/apm-21-2830 


\section{Introduction}

Stroke is a major cause of disability and mortality worldwide (1). China bears the largest stroke burden in the world, and the burden is expected to increase further as a result of the aging population (2-4). Over $80 \%$ of strokes occur in elderly individuals, and a patient's prognosis after stroke is vastly impacted by age (5). Despite the increasing proportion of elderly individuals in China, limited data are available on the clinical characteristics and prognosis of elderly patients. Identifying the profile of elderly stroke patients is valuable for developing specific secondary prevention and treatment strategies for the elderly. Several studies were previously conducted in Western populations, and studies on prognosis were scarce $(6,7)$. A previous study conducted approximately ten years ago indicated that compared with the Caucasian population, elderly stroke patients in China have a poor clinical prognosis, especially in terms of death and recurrence rates (8). Additionally, significant advancements have recently been made in the acute management and secondary prevention of acute ischaemic stroke (AIS), including the development of dual antiplatelet therapy and improved guidelines for thrombolysis and thrombectomy. Therefore, limited data are available on the current clinical characteristics, medication and prognosis of the oldest-old population, especially in China.

In this study, based on the Third China National Stroke Registry (CNSR-III), we aimed to evaluate the clinical characteristics, aetiology classification, medication persistence, and prognosis of oldest-old patients with ischaemic stroke or transient ischaemic attack (TIA) from 2015-2018 in China (9). We present the following article in accordance with the STROBE reporting checklist (available at https://apm.amegroups.com/article/view/10.21037/apm$21-2830 / \mathrm{rc})$.

\section{Methods}

\section{Data derivation and study population}

The data from this study were derived from CNSRIII, which is a prospective national registry that enrolled consecutive patients from 169 hospitals between August 2015 and March 2018 in China. There are a total of 15,166 patients with AIS or TIA within 7 days from onset of symptoms to enrollment. AIS and TIA were diagnosed according to the WHO criteria and confirmed by magnetic resonance imaging (MRI) or computed tomography (10). The protocol and data have been previously described in detail (11-13). In this study, oldest-old individuals were defined as $\geq 80$ years old.

\section{Clinical data collection}

The baseline data were obtained by standard trained researchers according to a typical protocol and using an electronic data capture system by face-to-face interviews. Baseline data included demographics (age, sex, living status, marital status, education, and insurance), medical history (prior hypertension, diabetes mellitus, stroke, TIA, coronary heart disease, heart failure and atrial fibrillation), smoking and drinking status, pre-stroke modified Rankin Scale (mRS), type of index event, National Institutes of Health Stroke Scale (NIHSS) score at admission, body mass index (BMI), blood pressure and medication use.

\section{Assessment of secondary prevention medication persistence}

In our study, persistent medication was defined as the continuation of secondary prevention medication from discharge to one year after the onset of symptoms. Patients assigned to a specific medication at discharge but who later discontinued the medication within one year were considered "nonpersistent". The same definition of persistence was provided for medication classes (e.g., antiplatelet, anticoagulant, lipid-lowering, antihypertensive, and antidiabetic medication). Patients were considered persistent if they discontinued one medication but took another medication of the same class within a year. Composite persistence was determined as the proportion ( $0 \%$ to $100 \%)$ of patients still taking the discharge medication at one year (14). Patients were classified into 3 groups based on compound persistence (level I: persistence $=0 \%$; level II: $0 \%<$ persistence $<100 \%$; and level III: persistence $=100 \%$ ).

\section{Imaging data collection}

Patients without MRI contradictions underwent a standard brain MRI scan on a 3.0 T or 1.5 T MRI scanner according to the standard protocol during hospitalization (11). The sequences and parameters of MRI scanning in this study have been reported (11). Image data were analysed centrally by trained neuroradiologists and the imaging data were interpreted with standardized forms in the electronic data capture (EDC) system, detailing the existence of infarction, infarction locations, and the relevance of arterial stenosis 
and index AIS or TIA.

The infarction number was evaluated in the diffusion weighted imaging (DWI) sequence. Infarction numbers were classified as no acute infarction (NAI), single acute infarction (SAI), and multiple acute infarctions (MAIs). Acute infarction was diagnosed as a visible hyperintense lesion on the DWI sequence. MAI was defined as more than one mutually "topographically distinct" lesion (spatially separated or discrete on consecutive slices). SAI was defined as unbroken lesions visualized in consecutive territories. Symptomatic intracranial/extracranial atherosclerotic stenosis (ICAS/ECAS) was diagnosed based on the presence of $\geq 50 \%$ stenosis of the vessel in the territory of the symptomatic vessel in a patient with TIA or stroke, based on the Warfarin-Aspirin Symptomatic Intracranial Disease Study (WASID) criteria (15), and the North American Symptomatic Carotid Endarterectomy Trial criteria for intracranial and extracranial arteries, respectively (16).

Images of all patients were interpreted by two neuroradiologists simultaneously blinded to the patients' clinical symptoms. Any disagreements were resolved by a third neuroradiologist.

\section{Aetiology classification}

Aetiological classification of ischaemic stroke was classified according to the Trial of Org 10172 in Acute Stroke Treatment (TOAST) criteria (17). Centralized stroke subtype was determined by neurologists and radiologists according to the previous defined standardized phenotypic element of each subtype (18). Each patient was interpreted by two neurologists or radiologists blinded to each other's input information on the standardized online screening report form generated from EDC. Discrepancies between the two forms were resolved by a third analyser.

\section{Follow-up and outcome evaluation}

Patients were followed up by telephone to obtain clinical outcomes at one year. Information including cardiovascular or cerebrovascular events and compliance with recommended secondary prevention medication was collected at follow-up. We recorded all stroke recurrence, combined vascular events, mortality, and poor functional outcomes during follow-up. Recurrent stroke, was defined as the new occurrence of focal neurological deficits caused by ischaemic or haemorrhagic stroke events and confirmed by MRI or computed tomography. Combined vascular events are defined as the new occurrence of stroke, myocardial infarction, and cardiovascular death. Poor functional outcome was defined as a score of 3-6 on the mRS. Cerebrovascular events were confirmed by the treating hospital and suspected recurrent cerebrovascular events without hospitalization were judged by an independent end point judgement committee. Death was either confirmed on a death certificate from the attended hospital or the local citizen registry.

\section{Ethical statement}

The study was conducted in accordance with the Declaration of Helsinki (as revised in 2013). The CNSR-III was approved by the ethics committee at Beijing Tiantan Hospital (IRB approval number: KY2015-001-01) and all participating centres. All patients provided written informed consent.

\section{Statistical analysis}

Patients were categorized into three groups: $<65,65-79$, and $\geq 80$ years. Continuous variables are presented as the median and interquartile range (IQR), while categorical variables are described as frequencies and proportions. Baseline characteristics were compared in patients of different age groups using chi-square tests for categorical variables and analysis of variance (ANOVA) or Kruskal-Wallis tests for continuous variables. Kaplan-Meier survival analysis was performed to calculate the one-year risk of stroke recurrence in AIS patients in various age groups. We used the multivariable Cox proportional hazards and logistic regression model (adjusted for sex, living alone, education, marital status, prestroke mRS 2-5, index event, NIHSS at admission, BMI, blood pressure, medical history of hypertension, diabetes mellitus, stroke, TIA, coronary artery disease, heart disease, arterial fibrillation, current smoker, heavy drinker, medication use at discharge, composite persistence and TOAST classification obtained from univariable analysis) to estimate the association between age and prognosis in all patients. Trend tests of prognosis across the different age groups of were conducted by Cochran-Armitage tend tests. We used SAS 9.4 software (SAS Institute, Inc., Cary, NC, USA) to perform all the statistical analyses, and a two-sided $\mathrm{P}$ value of less than 0.05 was considered significant.

\section{Results}

A total of 15,166 patients with AIS or TIA in CNSR-III 
were included in this study. Of them, 9,266 (61.10\%) were 65 years or younger, and $929(6.13 \%)$ were 80 years or older. Comparisons of demographic characteristics, social status, clinical presentation, and aetiology classification, and neuroimaging features by age are shown in Table 1 .
In comparison to nonelderly patients, oldest-old patients were more likely to be female, live alone, and have poorer education. In terms of stroke risk factors, oldest-old patients have a higher ratio of cardiac risk factors including medical history of atrial fibrillation, coronary artery

Table 1 Baseline characteristic of study participants

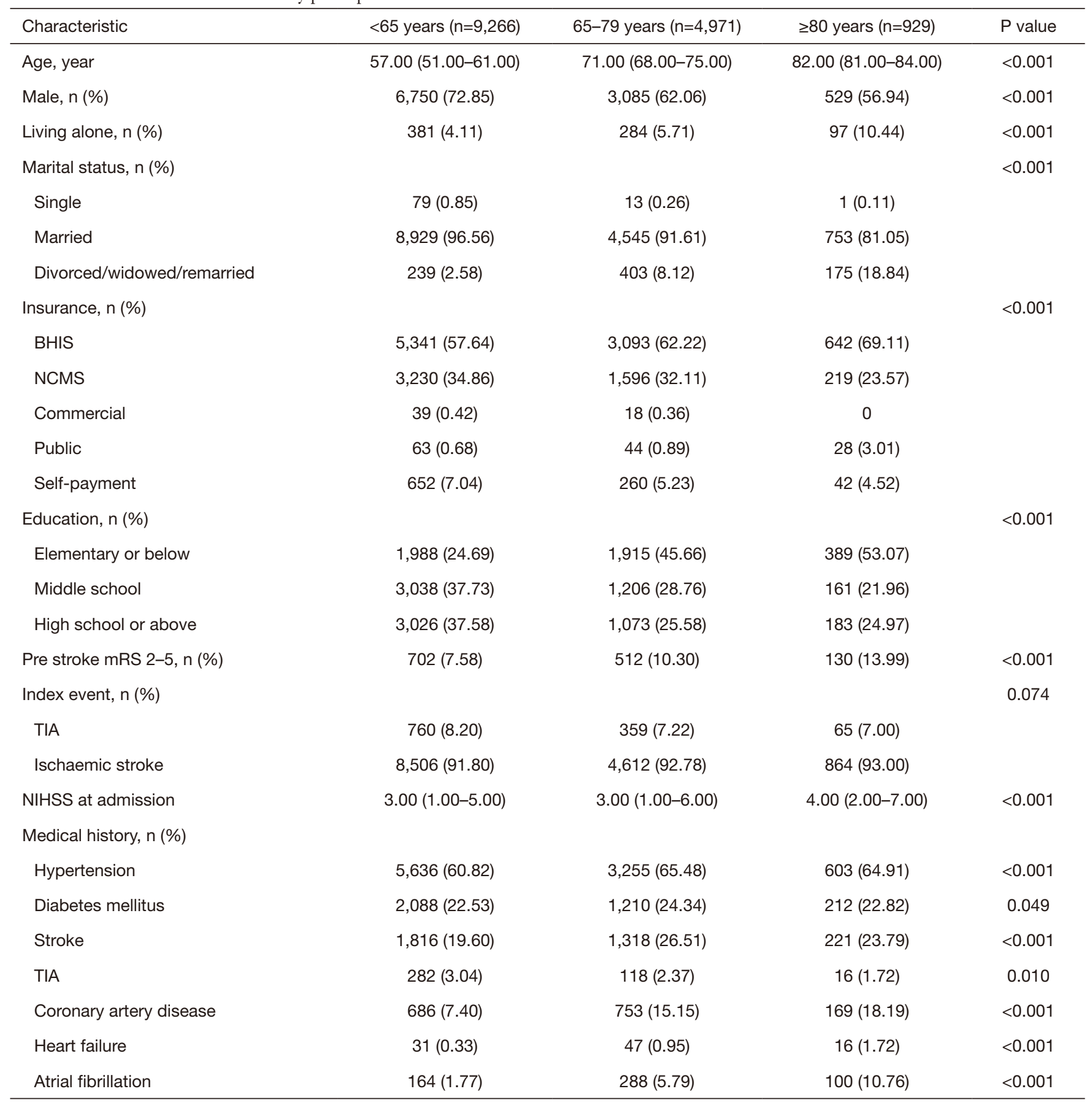

Table 1 (continued) 
Table 1 (continued)

\begin{tabular}{|c|c|c|c|c|}
\hline Characteristic & $<65$ years $(n=9,266)$ & $65-79$ years $(n=4,971)$ & $\geq 80$ years $(n=929)$ & $P$ value \\
\hline Heavy drinker, n (\%) & $1,612(17.40)$ & $469(9.43)$ & $45(4.84)$ & $<0.001$ \\
\hline Body mass index, $\mathrm{kg} / \mathrm{m}^{2}$ & 24.77 (22.92-26.83) & $24.22(22.22-26.12)$ & $23.44(21.20-25.71)$ & $<0.001$ \\
\hline Systolic blood pressure, mmHg & $147.50(134.50-163.00)$ & $149.00(135.00-164.00)$ & $149.00(137.00-164.50)$ & 0.011 \\
\hline Treated with rt-PA, n (\%) & $782(8.44)$ & $454(9.13)$ & $67(7.21)$ & 0.112 \\
\hline \multicolumn{5}{|l|}{ Medication use at discharge, n (\%) } \\
\hline Antiplatelet & $8,511(91.85)$ & 4,404 (88.59) & $796(85.68)$ & $<0.001$ \\
\hline Clopidogrel with Aspirin & $2,950(31.84)$ & $1,418(28.53)$ & $188(20.24)$ & $<0.001$ \\
\hline Antidiabetic agents & $2,180(23.57)$ & $1,184(23.87)$ & $182(19.74)$ & 0.022 \\
\hline Lipid-lowing agent & $8,472(91.43)$ & $4,521(90.95)$ & $838(90.20)$ & 0.340 \\
\hline Statin & 8,449 (91.18) & $4,513(90.79)$ & $837(90.10)$ & 0.455 \\
\hline Infection, n (\%) & $393(4.24)$ & $466(9.37)$ & $164(17.65)$ & $<0.001$ \\
\hline Composite persistence, $\mathrm{n}(\%)$ & & & & $<0.001$ \\
\hline Level I & $1,887(21.33)$ & $1,094(23.13)$ & $254(29.06)$ & \\
\hline Level II & $935(10.57)$ & $491(10.38)$ & $91(10.41)$ & \\
\hline Level III & 6,023 (68.09) & $3,144(66.48)$ & $529(60.53)$ & \\
\hline Undetermined etiology & $4,424(47.74)$ & $2,207(44.40)$ & $415(44.67)$ & \\
\hline Positive DWI lesion, n (\%) & $6,971(87.94)$ & $3,768(87.65)$ & $684(87.02)$ & 0.712 \\
\hline Infarction number, n (\%) & & & & $<0.001$ \\
\hline No infarction & $989(12.18)$ & $528(12.16)$ & $103(12.96)$ & \\
\hline Single acute infarction & $3,664(45.12)$ & $1,808(41.65)$ & $287(36.10)$ & \\
\hline Multiple acute infarctions & $3,467(42.70)$ & $2,005(46.19)$ & 405 (50.94) & \\
\hline Symptomatic ICAS, n (\%) & $2,107(25.95)$ & $1,253(28.86)$ & $291(36.60)$ & $<0.001$ \\
\hline Symptomatic ECAS, n (\%) & $378(4.66)$ & $291(6.70)$ & $53(6.67)$ & $<0.001$ \\
\hline
\end{tabular}

BHIS, Basic Health Insurance Scheme; NCMS, New Cooperative Medical System; mRS, modified Rankin Scale; TIA, transient ischaemic attack; NIHSS, National Institutes of Health Stroke Scale; rt-PA, recombinant tissue plasminogen activator; TOAST, Trial of Org 10172 in Acute stroke treatment; DWI, diffusion weighted imaging; ICAS, intracranial artery atherosclerosis; ECAS, extracranial atherosclerotic stenosis. 
Table 2 One-year prognosis of patients with acute ischaemic stroke or TIA by age

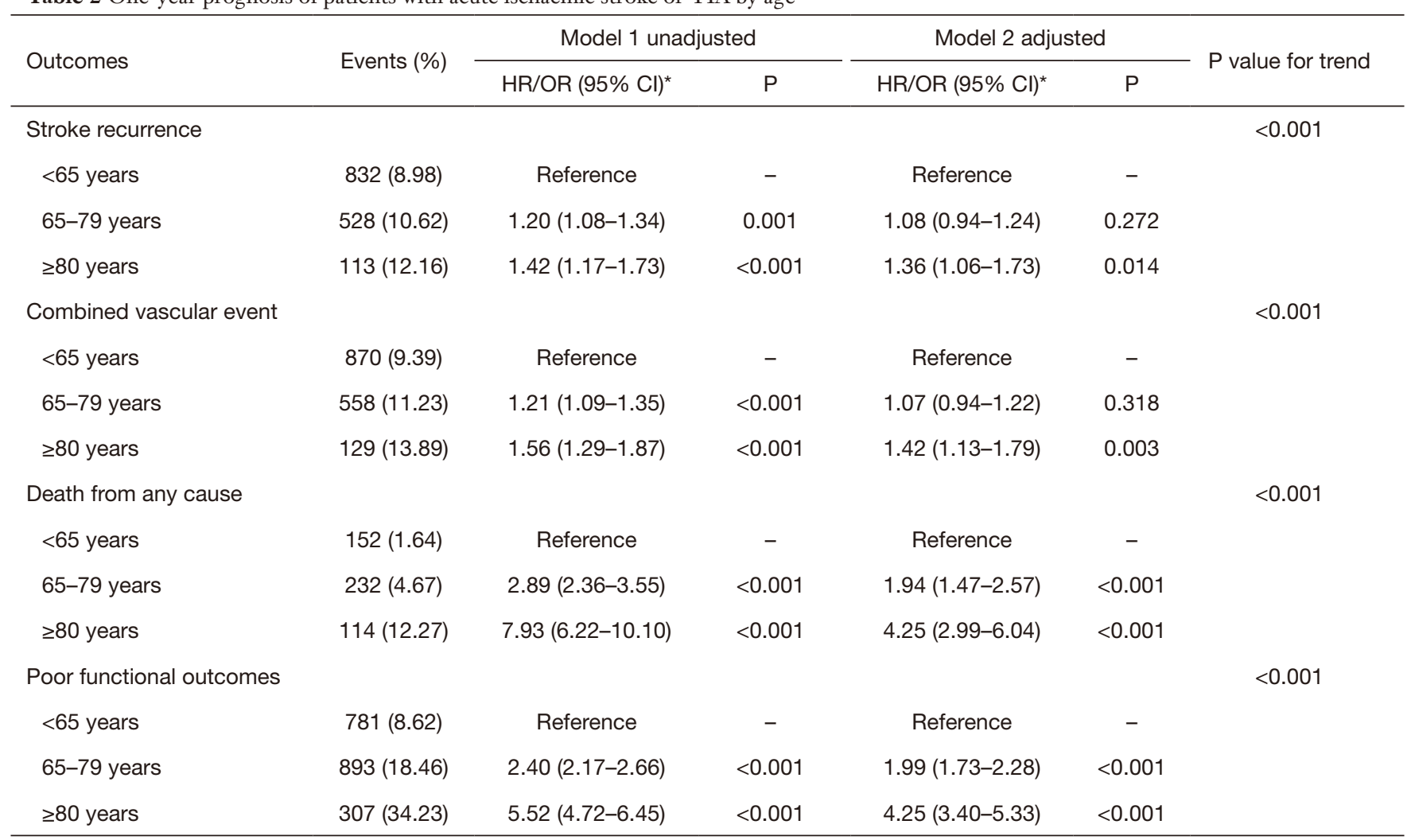

*, hazard ratio was used for the outcome of stroke recurrence, combined vascular event and death from any cause, odd ratios for the outcome of poor functional outcomes. Adjusted for: sex, living alone, education, marital status, pre stroke mRS 2-5, index event, NIHSS at admission, body mass index, systolic blood pressure, diastolic blood pressure, medical history of hypertension, diabetes mellitus, stroke, TIA, coronary artery disease, heart disease, arterial fibrillation, current drinking, heavy drinker, medication use of at discharge (antiplatelet, anticoagulation, antihypertensive, lipid-lowing agent), composite persistence, TOAST classification. TIA, transient ischaemic attack; OR, odds ratio; $\mathrm{HR}$, hazard ratio; $\mathrm{Cl}$, confidence interval.

disease, and heart failure. Medical history of hypertension, hyperlipidaemia, diabetes mellitus, and stroke were more likely to be observed in the 65-79-year group. Smoking and alcohol consumption are more frequently seen in the nonelderly population (under the age of 65).

At one year after symptom onset, in the oldest-old stroke patients, $60.53 \%$ of the study population took $100 \%$ of the medications prescribed for discharge (level III) and $29.06 \%$ took $0 \%$ (level I). The proportion of the composite persistence of level III in the elderly group, especially the oldest-old group, was higher than that in the nonelderly group.

Stroke aetiology based on TOAST classification by age is presented in Table 1. Stroke caused by large-artery atherosclerosis was still the most frequent in all age groups, except for undetermined aetiology. In addition, the proportion of cardioembolic stroke increased as age increased. Although there were no significant disparities in the proportion of DWI positivity among the different age groups, MAIs were more frequent in the oldest-old. Additionally, we compared the baseline characteristics of oldest-old and non-oldest-old adults ( $<80 v s$. $\geq 80$ years), and the results are similar with the baseline of Table 1 (Table S1).

\section{One-year prognosis after ischaemic stroke or TIA}

Table 2 shows the one-year prognosis at different ages. During the one-year follow-up, there were $113(12.16 \%)$ recurrent strokes, 129 (13.89\%) combined vascular events, and $307(34.23 \%)$ poor functional outcomes (mRS 3-6) in oldest-old patients.

In all patients with AIS, after adjustment for potential confounders in the univariable analysis (including sex, living alone, education, marital status, pre stroke mRS 2-5, index 


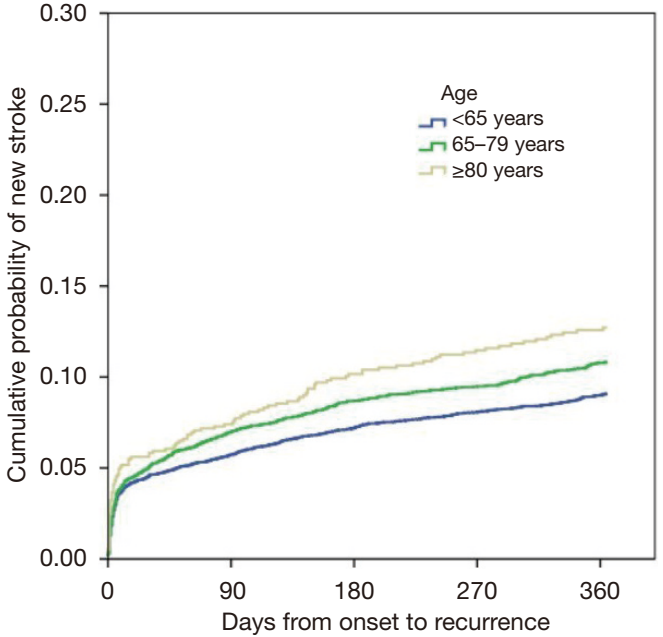

Figure 1 Kaplan-Meier event curves for one-year stroke recurrence by age.

event, NIHSS at admission, BMI, blood pressure, medical history and other potential confounders with $\mathrm{P}<0.05$ ), the oldest-old group patients had a significantly higher risk of stroke recurrence [hazard ratio (HR), 1.36; 95\% confidence interval (CI), 1.06-1.73], combined vascular events (HR, 1.42; 95\% CI, 1.13-1.79), mortality [odds ratio (OR), 4.25; 95\% CI, 2.99-6.04] and poor functional outcome (OR, 4.25; 95\% CI, 3.40-5.33). An increasing trend was observed in the risk of clinical outcomes including stroke recurrence, combined vascular events, mortality, and poor functional outcome as age increased ( $\mathrm{P}$ for trends $<0.001$, Table 2). Furthermore, the Kaplan-Meier curve shown in Figure 1 estimated the cumulative risk of one-year stroke recurrence by age.

\section{Discussion}

In this study, we found that the oldest-old patients were different from other patients regarding clinical characteristics, stroke aetiology, and secondary medication persistence in China. Oldest-old patients had the worst clinical prognosis.

Based on our CNSR-III data, the proportion of oldestold patients observed in our study was $6 \%$, similar to a previous study in China. In addition, we found that oldestold patients had more cardiovascular risk factors, such as a medical history of atrial fibrillation, coronary artery disease, and heart failure. Risk factors or smoking and drinking were more prevalent in patients aged $<65$ years. The medical history of hypertension, hyperlipidaemia, diabetes mellitus, and stroke were more common in the 65-79-year group, than in the oldest-old group. The age distribution and agespecific distribution of risk factors aligned with the CNSR-I data reported nearly a decade ago and did not change significantly (8). However, the proportion of oldest-old patients observed in our study was much lower than that in Western countries $(6,8)$. The lower proportion of oldest-old patients in this study may be partially attributed to the onset of ischaemic stroke at a younger age and the relatively lower life expectancy in China. the average age of stroke patients in China is almost 10 years younger than that in Western populations (19).

Regarding stroke aetiology, we found significantly more cases of cardioembolic stroke in the oldest-old compared with other age groups, which is consistent with previous studies $(20,21)$. This growing phenomenon can be explained by the increased proportion of atrial fibrillation in the older population, which is similar to previous studies $(9,22)$. The Framingham study also identified atrial fibrillation as most prevalent in elderly adults and the risk of stroke attributed to atrial fibrillation increased significantly with age, from $1.5 \%$ at ages $50-59$ years to $23.5 \%$ at ages $80-89$ years (23). However, compared with Western countries, the proportion of cardioembolic stroke in our study was much lower, which is consistent with previous studies $(7,24)$. The proportions of stroke subtypes are known to differ according to race and ethnicity: emboli originating from the heart are common in Western populations, whereas large-artery atherosclerosis is more prevalent in Asian populations (25). In addition, since we only considered cardioembolic aetiology with known atrial fibrillation and those diagnosed in early etiologic diagnostic workups, the results were most likely an underestimation of the true rate.

In this study, we identified that oldest-old patients had the worst prognosis including an increased risk of stroke recurrence, combined vascular events, poor functional outcome, and mortality at the one-year follow-up. Previous studies in Western countries have already found that very old age is a strong and independent predictor of mortality and early poor functional outcome after stroke, which is consistent with our study (8,26-29). For example, the Registry of the Canadian Stroke Network (RCSN) demonstrated that stroke fatality increased with age, with the highest 1 -year risk-adjusted fatality of $29.4 \%$ for oldestold patients compared to their younger counterparts. However, limited data have been reported on stroke recurrence in stroke patients in Western countries. In 
the present study, we obtained similar findings in stroke recurrence and combined vascular events in China, which is also consistent with 10-year-old data in China (8).

In addition to age as an unchangeable factor, increased risk factors as essential contributors and the presence of significantly more cardioembolic and large-artery atherosclerotic stroke types in the oldest-old may explain the poor prognosis in oldest-old stroke patients. Because cardioembolic strokes are related to a high risk of mortality and disability (30-32). And large-artery atherosclerotic stroke is associated with stroke recurrence $(32,33)$. Third, poor secondary prevention medication persistence in the oldest-old, especially antiplatelet and dual antibiotic therapy, may also to some extent explain poor prognosis. Previous studies have reported that medication persistence was associated with a reduced risk of recurrent stroke in patients with AIS or TIA (34). In addition, we found that the use of antiplatelet medication, especially dual antiplatelet medication, was lower in the elderly compared with other groups. The reason for this phenomenon may be because the current guidelines for stroke treatment recommending the use of antiplatelet agents were mainly based on populations under 80 years of age. In recent trials on dual versus single antiplatelet therapy, the proportions of patients aged $>80$ years were small, as the median age of patients included in the CHANCE, POINT and THALES trials was 63, 65, and 65 years, respectively (35-37). Due to inappropriate age limitations in randomized controlled trials and the almost universal exclusion of frail elderly people from studies, the evidence base for the oldest-old is inadequate (38). In addition, the uncertain balance of risks and benefits for antiplatelet therapy for the prevention of stroke in oldest-old individuals may also contribute to insufficient antiplatelet therapy $(38,39)$. Due to poor prognosis in the oldest-old, future RCTs avoiding upper age limits and standard exclusions are warranted.

When compared with the CNSR-I data reported almost ten years ago, our rate of stroke recurrence, mortality, and disability in the oldest-old was significantly reduced $36.02 \%$ vs. $12.16 \%, 34.78 \%$ vs. $12.27 \%, 43.81 \%$ vs. $20.77 \%$, respectively) (8). We consider that this phenomenon might be attributed to the universal acute management and improvement in secondary prevention in patients. Despite this, however, poor prognosis for patients of different ages is still a reminder that specific strategies to improve prognosis, such as increasing medication persistence, should be developed for patients of the secondary stroke prevention in oldest-old patients.
The strengths of our study include that the data for this study were recently developed from a large-scale, nationwide, prospective, multicentre cohort in China. In this study, we show the long-term outcomes of stroke patients in an oldest-old Asian population. Various outcomes, including stroke recurrence, combined vascular events, mortality and functional outcomes are described in this paper. However, our study also has limitations. First, most of the sites selected in this study were hospitals with more medical facilities and expertise than the lower-level hospitals that were excluded from the study, therefore, selection bias was inevitable. Second, insufficient information on endovascular thrombectomy therapy was available for oldest-old stroke patients in this study.

In conclusion, the oldest-old patients were different from their younger counterparts in clinical characteristics, stroke aetiology, and secondary medication persistence. In addition, although the prognosis of the oldest-old had improved compared with the CNSR-I study reported previously, it was still the worst in different age groups of ischaemic stroke and TIA in China. The poor prognosis of the oldest-old might be partially attributed to the high proportion of cardioembolism and large-artery atherosclerosis-associated strokes in TOAST classification, and lower secondary preventive medication persistence. Future treatment strategies and specialized care facilities in oldest-old patients should be seriously considered to improve secondary prevention and prognosis in oldest-old stroke patients (40).

\section{Acknowledgments}

We thank all the participants in the study.

Funding: This work was supported by grants from National Key R\&D Program of China (2018YFC1312903), grants from the National Natural Science Foundation of China (81870905), grants from Beijing Municipal Science \& Technology Commission (D171100003017002, Z181100001818001).

\section{Footnote}

Reporting Checklist: The authors have completed the STROBE reporting checklist. Available at https://apm. amegroups.com/article/view/10.21037/apm-21-2830/rc

Data Sharing Statement: Available at https://apm.amegroups. 
com/article/view/10.21037/apm-21-2830/dss

Conflicts of Interest: All authors have completed the ICMJE uniform disclosure form (available at https://apm. amegroups.com/article/view/10.21037/apm-21-2830/coif). The authors have no conflicts of interest to declare.

Ethical Statement: The authors are accountable for all aspects of the work in ensuring that questions related to the accuracy or integrity of any part of the work are appropriately investigated and resolved. The study was conducted in accordance with the Declaration of Helsinki (as revised in 2013). The CNSR-III was approved by the ethics committee at Beijing Tiantan Hospital (IRB approval number: KY2015-001-01) and all participating centres. All patients provided written informed consent.

Open Access Statement: This is an Open Access article distributed in accordance with the Creative Commons Attribution-NonCommercial-NoDerivs 4.0 International License (CC BY-NC-ND 4.0), which permits the noncommercial replication and distribution of the article with the strict proviso that no changes or edits are made and the original work is properly cited (including links to both the formal publication through the relevant DOI and the license). See: https://creativecommons.org/licenses/by-nc-nd/4.0/.

\section{References}

1. GBD 2019 Diseases and Injuries Collaborators. Global burden of 369 diseases and injuries in 204 countries and territories, 1990-2019: a systematic analysis for the Global Burden of Disease Study 2019. Lancet 2020;396:1204-22.

2. Wu S, Wu B, Liu M, et al. Stroke in China: advances and challenges in epidemiology, prevention, and management. Lancet Neurol 2019;18:394-405.

3. Fang EF, Xie C, Schenkel JA, et al. A research agenda for ageing in China in the 21 st century (2nd edition): Focusing on basic and translational research, long-term care, policy and social networks. Ageing Res Rev 2020;64:101174.

4. Wang W, Jiang B, Sun H, et al. Prevalence, Incidence, and Mortality of Stroke in China: Results from a Nationwide Population-Based Survey of 480687 Adults. Circulation 2017;135:759-71.

5. Chen RL, Balami JS, Esiri MM, et al. Ischemic stroke in the elderly: an overview of evidence. Nat Rev Neurol 2010;6:256-65.

6. Saposnik G, Black SE, Hakim A, et al. Age disparities in stroke quality of care and delivery of health services. Stroke 2009;40:3328-35.

7. Béjot $Y$, Duloquin G, Graber M, et al. Current characteristics and early functional outcome of older stroke patients: a population-based study (Dijon Stroke Registry). Age Ageing 2021;50:898-905.

8. Deng YX, Wang YL, Gao BQ, et al. Age differences in clinical characteristics, health care, and outcomes after ischemic stroke in China. CNS Neurosci Ther 2012;18:819-26.

9. Lindley RI. Stroke Prevention in the Very Elderly. Stroke 2018;49:796-802.

10. Stroke--1989. Recommendations on stroke prevention, diagnosis, and therapy. Report of the WHO Task Force on Stroke and other Cerebrovascular Disorders. Stroke 1989;20:1407-31.

11. Wang Y, Jing J, Meng X, et al. The Third China National Stroke Registry (CNSR-III) for patients with acute ischaemic stroke or transient ischaemic attack: design, rationale and baseline patient characteristics. Stroke Vasc Neurol 2019;4:158-64.

12. Jing J, Suo Y, Wang A, et al. Imaging Parameters Predict Recurrence After Transient Ischemic Attack or Minor Stroke Stratified by ABCD2 Score. Stroke 2021;52:2007-15.

13. Xu J, Yalkun G, Wang M, et al. Impact of Infection on the Risk of Recurrent Stroke Among Patients With Acute Ischemic Stroke. Stroke 2020;51:2395-403.

14. Bushnell CD, Olson DM, Zhao X, et al. Secondary preventive medication persistence and adherence 1 year after stroke. Neurology 2011;77:1182-90.

15. Samuels OB, Joseph GJ, Lynn MJ, et al. A standardized method for measuring intracranial arterial stenosis. AJNR Am J Neuroradiol 2000;21:643-6.

16. North American Symptomatic Carotid Endarterectomy Trial Collaborators; Barnett HJM, Taylor DW, et al. Beneficial effect of carotid endarterectomy in symptomatic patients with high-grade carotid stenosis. N Engl J Med 1991;325:445-53.

17. Adams HP Jr, Bendixen BH, Kappelle LJ, et al. Classification of subtype of acute ischemic stroke. Definitions for use in a multicenter clinical trial. TOAST. Trial of Org 10172 in Acute Stroke Treatment. Stroke 1993;24:35-41.

18. Suo Y, Jing J, Meng X, et al. Inconsistent centralised versus non-centralised ischaemic stroke aetiology. Stroke Vasc Neurol 2020;5:337-47.

19. Li Z, Jiang $\mathrm{Y}, \mathrm{Li} \mathrm{H}$, et al. China's response to the rising stroke burden. BMJ 2019;364:1879. 
20. Kato Y, Hayashi T, Tanahashi N, et al. Cardioembolic stroke is the most serious problem in the aging society: Japan standard stroke registry study. J Stroke Cerebrovasc Dis 2015;24:811-4.

21. Gur AY, Tanne D, Bornstein NM, et al. Stroke in the very elderly: characteristics and outcome in patients aged $\geq 85$ years with a first-ever ischemic stroke. Neuroepidemiology 2012;39:57-62.

22. Yiin GSC, Li L, Bejot Y, et al. Time Trends in Atrial Fibrillation-Associated Stroke and Premorbid Anticoagulation. Stroke 2018. [Epub ahead of print]. doi: 10.1161/STROKEAHA.118.022249.

23. Wolf PA, Abbott RD, Kannel WB. Atrial fibrillation as an independent risk factor for stroke: the Framingham Study. Stroke 1991;22:983-8.

24. Hart RG, Diener HC, Coutts SB, et al. Embolic strokes of undetermined source: the case for a new clinical construct. Lancet Neurol 2014;13:429-38.

25. Kim BJ, Kim JS. Ischemic stroke subtype classification: an asian viewpoint. J Stroke 2014;16:8-17.

26. Sanossian N, Apibunyopas KC, Liebeskind DS, et al. Characteristics and Outcomes of Very Elderly Enrolled in a Prehospital Stroke Research Study. Stroke 2016;47:2737-41.

27. Russo T, Felzani G, Marini C. Stroke in the very old: a systematic review of studies on incidence, outcome, and resource use. J Aging Res 2011;2011:108785.

28. Saposnik G, Cote R, Phillips S, et al. Stroke outcome in those over 80: a multicenter cohort study across Canada. Stroke 2008;39:2310-7.

29. Kammersgaard LP, Jørgensen HS, Reith J, et al. Shortand long-term prognosis for very old stroke patients. The Copenhagen Stroke Study. Age Ageing 2004;33:149-54.

30. Ferro JM. Cardioembolic stroke: an update. Lancet

Cite this article as: Wang Y, Jing J, Pan Y, Wang M, Meng X, Wang Y. Clinical characteristics and prognosis in oldest old patients with ischaemic stroke or transient ischaemic attack in China. Ann Palliat Med 2022;11(7):2215-2224. doi: 10.21037/apm21-2830
Neurol 2003;2:177-88.

31. Arsava EM, Helenius J, Avery R, et al. Assessment of the Predictive Validity of Etiologic Stroke Classification. JAMA Neurol 2017;74:419-26.

32. Petty GW, Brown RD Jr, Whisnant JP, et al. Ischemic stroke subtypes : a population-based study of functional outcome, survival, and recurrence. Stroke 2000;31:1062-8.

33. Amarenco P, Lavallée PC, Labreuche J, et al. One-Year Risk of Stroke after Transient Ischemic Attack or Minor Stroke. N Engl J Med 2016;374:1533-42.

34. Zhang L, Shi J, Pan Y, et al. Secondary prevention medication persistence and prognosis of acute ischaemic stroke or transient ischaemic attack. Stroke Vasc Neurol 2021;6:376-83.

35. Johnston SC, Easton JD, Farrant M, et al. Clopidogrel and Aspirin in Acute Ischemic Stroke and High-Risk TIA. N Engl J Med 2018;379:215-25.

36. Wang Y, Wang Y, Zhao X, et al. Clopidogrel with aspirin in acute minor stroke or transient ischemic attack. N Engl J Med 2013;369:11-9.

37. Kaushik A, Deora S, Choudhary R. Ticagrelor and Aspirin or Aspirin Alone in Acute Ischemic Stroke or TIA. N Engl J Med 2020;383:1692-3.

38. Lindley RI. Drug trials for older people. J Gerontol A Biol Sci Med Sci 2012;67:152-7.

39. Li L, Geraghty OC, Mehta Z, et al. Age-specific risks, severity, time course, and outcome of bleeding on longterm antiplatelet treatment after vascular events: a population-based cohort study. Lancet 2017;390:490-9.

40. Marcum ZA, Hanlon JT, Murray MD. Improving Medication Adherence and Health Outcomes in Older Adults: An Evidence-Based Review of Randomized Controlled Trials. Drugs Aging 2017;34:191-201. 
Table S1 Baseline characteristic of study participants, in oldest-old and non-oldest-old patients

\begin{tabular}{|c|c|c|c|}
\hline Characteristic & $<80$ years $(n=14,237)$ & $\geq 80$ years $(n=929)$ & $P$ value \\
\hline Age, year & $62.00(54.00-68.00)$ & $82.00(81.00-84.00)$ & $<0.001$ \\
\hline Male, n (\%) & $9835(69.08)$ & $529(56.94)$ & $<0.001$ \\
\hline Living alone, n (\%) & $665(4.67)$ & $97(10.44)$ & $<0.001$ \\
\hline Marital status & & & $<0.001$ \\
\hline Single & $92(0.65)$ & $1(0.11)$ & \\
\hline Married & $13474(94.83)$ & $753(81.05)$ & \\
\hline Divorced/widowed/remarried & $642(4.52)$ & $175(18.84)$ & \\
\hline Insurance, n (\%) & & & $<0.001$ \\
\hline BHIS & $8434(59.24)$ & 642 (69.11) & \\
\hline NCMS & $4826(33.90)$ & $219(23.57)$ & \\
\hline Commercial & $57(0.40)$ & 0 & \\
\hline Public & $107(0.75)$ & $28(3.01)$ & \\
\hline Self-payment & $912(6.41)$ & $42(4.52)$ & \\
\hline Education, n (\%) & & & $<0.001$ \\
\hline Elementary or below & $3903(31.87)$ & $389(53.07)$ & \\
\hline Middle school & $4244(34.66)$ & $161(21.96)$ & \\
\hline High school or above & $4099(33.47)$ & $183(24.97)$ & \\
\hline Pre stroke mRS 2-5, n (\%) & $1214(8.53)$ & 130 (13.99) & $<0.001$ \\
\hline Index event, n (\%) & & & 0.342 \\
\hline TIA & $1119(7.86)$ & $65(7.00)$ & \\
\hline Ischaemic stroke & 13118 (92.14) & $864(93.00)$ & \\
\hline NIHSS at admission & $3.00(1.00-6.00)$ & $4.00(2.00-7.00)$ & $<0.001$ \\
\hline \multicolumn{4}{|l|}{ Medical history, n (\%) } \\
\hline Hypertension & $8891(62.45)$ & $603(64.91)$ & 0.134 \\
\hline Diabetes mellitus & $3298(23.16)$ & $212(22.82)$ & 0.809 \\
\hline Stroke & $3134(22.01)$ & 221 (23.79) & 0.206 \\
\hline TIA & $400(2.81)$ & $16(1.72)$ & 0.049 \\
\hline Coronary artery disease & $1439(10.11)$ & 169 (18.19) & $<0.001$ \\
\hline Heart failure & $78(0.55)$ & $16(1.72)$ & $<0.001$ \\
\hline Atrial fibrillation & $452(3.17)$ & $100(10.76)$ & $<0.001$ \\
\hline Current smoker, n (\%) & $4655(32.70)$ & $97(10.44)$ & $<0.001$ \\
\hline Heavy drinker, $\mathrm{n}(\%)$ & $2081(14.62)$ & $45(4.84)$ & $<0.001$ \\
\hline Body mass index & $24.49(22.72-26.57)$ & $23.44(21.20-25.71)$ & $<0.001$ \\
\hline Systolic blood pressure, mmHg & $148.00(135.00-163.50)$ & $149.00(137.00-164.50)$ & 0.130 \\
\hline Diastolic blood pressure, $\mathrm{mmHg}$ & $87.00(79.50-96.00)$ & $80.00(73.50-89.00)$ & $<0.001$ \\
\hline Treated with rt-PA, n (\%) & $1236(8.68)$ & $67(7.21)$ & 0.122 \\
\hline \multicolumn{4}{|l|}{ Medication use at discharge, $\mathrm{n}(\%)$} \\
\hline Antiplatelet & $12943(90.91)$ & $796(85.68)$ & $<0.001$ \\
\hline Clopidogrel with Aspirin & $4368(30.68)$ & $188(20.24)$ & $<0.001$ \\
\hline Anticoagulants & $430(3.02)$ & $47(5.06)$ & $<0.001$ \\
\hline Antihypertensive agent & $6966(48.93)$ & $480(51.67)$ & 0.039 \\
\hline Antidiabetic agents & $3364(23.68)$ & $182(19.74)$ & 0.006 \\
\hline Lipid-lowing agent & 13021 (91.46) & $838(90.20)$ & 0.597 \\
\hline Statin & $12962(91.04)$ & $837(90.10)$ & 0.329 \\
\hline Infection, $\mathrm{n}(\%)$ & $859(6.03)$ & $164(17.65)$ & $<0.001$ \\
\hline Composite persistence, $\mathrm{n}(\%)$ & & & $<0.001$ \\
\hline Level I & 2981 (21.96) & $254(29.06)$ & \\
\hline Level II & $1426(10.51)$ & $91(10.41)$ & \\
\hline Level III & $9167(67.53)$ & $529(60.53)$ & \\
\hline TOAST classification, n (\%) & & & $<0.001$ \\
\hline Large-artery atherosclerosis & $3606(25.33)$ & $250(26.91)$ & \\
\hline Cardioembolism & $785(5.51)$ & $132(14.21)$ & \\
\hline Small-artery occlusion & $3042(21.37)$ & $123(13.24)$ & \\
\hline Other determined etiology & $173(1.22)$ & $9(0.97)$ & \\
\hline Undetermined etiology & $6631(46.58)$ & $415(44.67)$ & \\
\hline Positive DWI lesion, n (\%) & $10739(87.84)$ & $684(87.02)$ & 0.499 \\
\hline Infarction number n (\%) & & & $<0.001$ \\
\hline No infarction & $1517(12.17)$ & $103(12.96)$ & \\
\hline Single acute infarction & $5472(43.91)$ & $287(36.10)$ & \\
\hline Multiple acute infarctions & $5472(43.91)$ & 405 (50.94) & \\
\hline Symptomatic ICAS & 3360 (26.96) & $291(36.60)$ & $<0.001$ \\
\hline Symptomatic ECAS & $669(5.37)$ & $53(6.67)$ & 0.118 \\
\hline
\end{tabular}

BHIS, Basic Health Insurance Scheme; NCMS, New Cooperative Medical System; mRS, modified Rankin Scale; NIHSS, National Institutes of Health Stroke Scale; TIA, transient ischaemic attack; rt-PA, recombinant tissue plasminogen activator; TOAST: Trial of Org 10172 in Acute stroke treatment; DWI, diffusion weighted imaging; ICAS, intracranial artery atherosclerosis; ECAS, extracranial atherosclerotic stenosis. 